TRANSACTIONS OF THE

AMERICAN MATHEMATICAL SOCIETY

Volume 359, Number 5, May 2007, Pages 2059-2074

S 0002-9947(06)04026-8

Article electronically published on December 15, 2006

\title{
GENERATING CONTINUOUS MAPPINGS WITH LIPSCHITZ MAPPINGS
}

\author{
J. CICHOŃ, J. D. MITCHELL, AND M. MORAYNE
}

\begin{abstract}
If $X$ is a metric space, then $\mathcal{C}_{X}$ and $\mathcal{L}_{X}$ denote the semigroups of continuous and Lipschitz mappings, respectively, from $X$ to itself. The relative rank of $\mathcal{C}_{X}$ modulo $\mathcal{L}_{X}$ is the least cardinality of any set $U \backslash \mathcal{L}_{X}$ where $U$ generates $\mathcal{C}_{X}$. For a large class of separable metric spaces $X$ we prove that the relative rank of $\mathcal{C}_{X}$ modulo $\mathcal{L}_{X}$ is uncountable. When $X$ is the Baire space $\mathbb{N}^{\mathbb{N}}$, this rank is $\aleph_{1}$. A large part of the paper emerged from discussions about the necessity of the assumptions imposed on the class of spaces from the aforementioned results.
\end{abstract}

\section{INTRODUCTION AND PRELIMINARIES}

Given a mathematical structure $X$ and a subset $Y$ of $X$, it is natural to ask how the 'size' of $Y$ compares to that of $X$. If $X$ and $Y$ are both infinite, there is no single obvious way of answering this question. In fact, a large part of mathematics is devoted to answering this type of question. For instance, in analysis and topology, two standard notions of size are measure and category. In this paper we consider an algebraic notion of size. Let $X$ be a semigroup and let $Y$ be a subset of $X$. The relative rank of $X$ modulo $Y$, denoted by $\operatorname{rank}(X: Y)$, is defined as the least cardinality of a subset $Z$ of $X$ such that $Y \cup Z$ generates $X$. We may also refer to the relative rank of $Y$ in $X$.

Relative ranks have been intensely investigated for semigroups of different types of mappings. For example, a classical result in the field, proved by Sierpiński, states that every countable set of mappings from an infinite set $X$ to itself can be generated using two such mappings; see [1], 7] or [12. The semigroup of all mappings from $X$ to $X$ is denoted by $\mathcal{T}_{X}$. As an immediate consequence of Sierpiński's result, it follows that any subset of $\mathcal{T}_{X}$ has relative rank $0,1,2$ or uncountably infinite. An important subset of $\mathcal{T}_{X}$ is, of course, the symmetric group on $X$, denoted by $\mathcal{S}_{X}$. Another early result says that $\operatorname{rank}\left(\mathcal{T}_{X}: \mathcal{S}_{X}\right)=2$; see [6] and [9]. In [5], to mention another interesting result, it has been shown that whenever $G \subseteq \mathcal{S}_{X}$ then $\operatorname{rank}\left(\mathcal{S}_{X}: G\right)$ is 0,1 or uncountable. Slight modifications of Banach's proof of Sierpiński's result can be used to prove analogous results for binary relations, partial mappings, and partial bijections of infinite sets; see [7.

Received by the editors January 28, 2005.

2000 Mathematics Subject Classification. Primary 54H15, $20 \mathrm{M} 20$.

Key words and phrases. Relative ranks, functions spaces, continuous mappings, Lipschitz mappings, Baire space. 
In this paper, we investigate the relative ranks of continuous mappings modulo Lipschitz mappings on certain metric spaces. For a metric space $X$, let $\mathcal{C}_{X}$ denote the semigroup of continuous mappings from $X$ to $X$ and let $\mathcal{L}_{X}$ denote the semigroup of Lipschitz mappings from $X$ to $X$. We show that $\operatorname{rank}\left(\mathcal{C}_{X}: \mathcal{L}_{X}\right)$ is uncountable for two large classes of metric spaces (Theorems 2.1 and 3.2). The fact that we considered these function spaces and the particular metric spaces on which they act, was, in part, motivated by connections to relative ranks of endomorphisms of ordered sets and graphs. In fact, the first of our results concerning $\operatorname{rank}\left(\mathcal{C}_{X}: \mathcal{L}_{X}\right)$ may be applied to endomorphisms of graphs and ordered sets (Corollaries 2.4 and 2.5).

Furthermore, in Sections 4 and 5 we give examples that demonstrate that none of the assumptions made about the metric spaces, that are the subject of Theorems 2.1 and 3.2. can be removed. Related to this discussion is Theorem 6.1, in which we determine the exact rank of $\mathcal{C}_{X}$ modulo $\mathcal{L}_{X}$, where $X$ is a space that plays an important role in descriptive set theory, the Baire space.

It is known that for many natural metric spaces the family of Lipschitz mappings is a meager subset of the space of all continuous functions with the topology of uniform convergence. Thus from a topological point of view $\mathcal{L}_{X}$ is 'small' in $\mathcal{C}_{X}$. Our main results say that the same is true, from an algebraic point of view, for some classes of metric space.

In the remainder of this section, we will give all the definitions and notation necessary for the rest of the paper.

As usual, for a semigroup $S$ and $U \subseteq S$, denote by $\langle U\rangle$ the subsemigroup generated by $U$. For brevity, if $U, V \subseteq S$, we write $\langle U, V\rangle$ instead of, the formally correct, $\langle U \cup V\rangle$.

A metric space $X$ is concentric if it is unbounded and it is the union of an increasing sequence of compact balls. Such spaces are common, for example every euclidean space $\mathbb{R}^{n}$ is concentric. The induced topology of a concentric metric space is noncompact, locally compact and second countable. On the other hand, for a topological space $(X, \mathcal{U})$ which is noncompact, locally compact and second countable there exists a metric $d$ such that $(X, d)$ is concentric; see [14].

Let $\sigma$ be a sequence in a metric space $X$ and let $Y$ be the set of elements that occur in $\sigma$, and its limit $l$, if it exists. Then $\sigma$ is said to be extendible if $Y$ is infinite and every continuous map from $Y$ to $Y$ that fixes $l$, if it exists, can be extended to an element of $\mathcal{C}_{X}$. A retract is a subset $R$ of a metric space that occurs as the image of a continuous mapping that acts as the identity on $R$. Throughout $\mathbb{N}<\mathbb{N}$

denotes the set of all finite sequences of natural numbers. An element of $\mathbb{N}<\mathbb{N}$ is denoted by $\vec{i}$, and $|\vec{i}|$ denotes the length of the sequence.

\section{Concentric SPACES}

In this section we prove that the relative rank of $\mathcal{C}_{X}$ modulo $\mathcal{L}_{X}$ is uncountable if $X$ is a concentric space satisfying a particular property. This is accomplished in the next theorem. At the end of the section, we discuss various corollaries to the main result of this section.

Theorem 2.1. Let $X$ be a concentric metric space which contains an extendible sequence with no convergent subsequence. Then $\operatorname{rank}\left(\mathcal{C}_{X}: \mathcal{L}_{X}\right)$ is uncountable. 
Proof. It suffices to prove that for every countable subset of $\mathcal{C}_{X}$ there exists a continuous mapping that is not generated by the union of the Lipschitz mappings and this subset. We construct such a mapping $\gamma$ recursively.

To this end, let $\mu_{1}, \mu_{2}, \ldots \in \mathcal{C}_{X}$ be arbitrary, let $\rho=\left(x_{0}, x_{1}, x_{2}, \ldots\right)$ be an extendible sequence with no convergent subsequence, and let $B(p, 1) \subseteq B(p, 2)$ $\subseteq \cdots$ be compact balls that comprise $X$ for some $p \in X$. Since all the balls we consider in this proof are centered on $p$, for brevity we write $B(n)$ instead of $B(p, n)$. The elements of $\left\langle\mathcal{L}_{X}, \mu_{1}, \mu_{2}, \ldots\right\rangle$ are finite compositions of the form

$$
\Phi_{m+1} \mu_{i_{m}} \cdots \Phi_{2} \mu_{i_{1}} \Phi_{1}
$$

for some $i_{1}, i_{2}, \ldots, i_{m} \in \mathbb{N}$ and $\Phi_{1}, \Phi_{2}, \ldots, \Phi_{m+1} \in \mathcal{L}_{X}$. We represent $\left\langle\mathcal{L}_{X}, \mu_{1}, \mu_{2}, \ldots\right\rangle$ as a countable union of families of such compositions. These families are determined, roughly speaking, according to the elements of $\left\{\mu_{1}, \mu_{2}, \ldots\right\}$ that appear, the Lipschitz constants involved, and the balls that contain images of $x_{0}$. The mapping $\gamma$ is initially defined on the sequence $\rho$. Thus there are countably many steps in the definition, at each of which we ensure that $\gamma$ does not belong to one of the given families. This process exhausts every possibility.

Precisely speaking, we consider the set

$$
\Sigma=\{[\vec{i}, n]: \vec{i} \in \mathbb{N}<\mathbb{N}, n \in \mathbb{N}\}
$$

and for $[\vec{i}, n] \in \Sigma$, with $\vec{i}=\left(i_{1}, i_{2}, \ldots, i_{k}\right)$ and $n \in \mathbb{N}$, the family of compositions

$$
\begin{aligned}
\mathcal{F}_{[\vec{i}, n]}=\left\{\Phi_{k+1} \mu_{i_{k}} \cdots \Phi_{2} \mu_{i_{1}} \Phi_{1}:\right. & \Phi_{j+1} \mu_{i_{j}} \Phi_{j} \mu_{i_{j-1}} \cdots \Phi_{2} \mu_{i_{1}} \Phi_{1}\left(x_{0}\right) \in B(n), \\
& \left.\Phi_{j+1} \in \mathcal{L}_{X} \text { with constant } n \text { for each } 0 \leq j \leq k\right\} .
\end{aligned}
$$

Obviously,

$$
\bigcup_{[\vec{i}, n] \in \Sigma} \mathcal{F}_{[\vec{i}, n]}=\left\langle\mathcal{L}_{X}, \mu_{1}, \mu_{2}, \ldots\right\rangle .
$$

Since $\Sigma$ is countable we may enumerate its elements as $\sigma_{1}, \sigma_{2}, \ldots$.

Step 0 in the definition of $\gamma$ is made by setting $\gamma\left(x_{0}\right)=x_{0}$.

At step $r>0$ we define $\gamma$ on $x_{r}$ in such a way that when the definition is complete no extension of $\gamma$ to an element of $\mathcal{C}_{X}$ is an element of $\mathcal{F}_{\sigma_{r}}$. Assume that $\gamma$ was defined on $x_{0}, x_{1}, \ldots, x_{r-1}$ in the previous steps such that any element of $\mathcal{C}_{X}$ agreeing with $\gamma$ on these points does not lie in $\mathcal{F}_{\sigma_{i}}$ for $i<r$. Also assume that $\sigma_{r}=[\vec{i}, n]$ with $\vec{i}=\left(i_{1}, \ldots, i_{k}\right)$ and $n \in \mathbb{N}$.

This step is completed by proving that every element of $\mathcal{F}_{\sigma_{r}}$ maps $x_{r}$ into the same ball $B(m)$ for some $m$. In fact, it suffices to prove that if for each $0 \leq j \leq k-1$ and $\Phi_{j+1}$ as in (2) we have $\Phi_{j+1} \mu_{i_{j}} \cdots \Phi_{2} \mu_{i_{1}} \Phi_{1}\left(x_{r}\right) \in B\left(m_{j+1}\right)$, for some $m_{j+1}$, then every $\Phi_{j+2} \mu_{i_{j+1}} \cdots \Phi_{2} \mu_{i_{1}} \Phi_{1}\left(x_{r}\right) \in B\left(m_{j+2}\right)$, for some $m_{j+2}$. Thus within step $r$ we perform a finite induction.

For the base case, $\Phi_{1}\left(x_{0}\right) \in B(n)$ and $d\left(\Phi_{1}\left(x_{0}\right), \Phi_{1}\left(x_{r}\right)\right) \leq n d\left(x_{0}, x_{r}\right)$. Therefore $d\left(\Phi_{1}\left(x_{r}\right), p\right) \leq n+n d\left(x_{0}, x_{r}\right)$, and so $\Phi_{1}\left(x_{r}\right) \in B\left(m_{1}\right)$, where $m_{1}=n+n d\left(x_{0}, x_{r}\right)$. Note that the choice of $m_{1}$ does not depend on $\Phi_{1}$, but on its Lipschitz constant.

The inductive hypothesis states that for every $\Phi_{j+1} \mu_{i_{j}} \cdots \Phi_{2} \mu_{i_{1}} \Phi_{1} \in \mathcal{F}_{\left[\left(i_{1}, \ldots, i_{j}\right), n\right]}$ we have

$$
u=\Phi_{j+1} \mu_{i_{j}} \cdots \Phi_{2} \mu_{i_{1}} \Phi_{1}\left(x_{r}\right) \in B\left(m_{j+1}\right)
$$

for some $m_{j+1}$. By the definition of $\mathcal{F}_{[\vec{i}, n]}$

$$
v=\Phi_{j+1} \mu_{i_{j}} \cdots \Phi_{2} \mu_{i_{1}} \Phi_{1}\left(x_{0}\right) \in B(n) .
$$


If $M$ is the maximum of $m_{j+1}$ and $n$, then $\mu_{i_{j+1}}(u), \mu_{i_{j+1}}(v) \in \mu_{i_{j+1}}(B(M))$. Since the continuous image of a compact set is compact, there exists $M^{\prime}$ such that $\mu_{i_{j+1}}(B(M)) \subseteq B\left(M^{\prime}\right)$. Thus $d\left(\mu_{i_{j+1}}(u), \mu_{i_{j+1}}(v)\right) \leq 2 M^{\prime}$. But, again by the definition of $\mathcal{F}_{[\vec{i}, n]}, \Phi_{j+2} \mu_{i_{j+1}}(v) \in B(n)$. Therefore

$$
d\left(p, \Phi_{j+2} \mu_{i_{j+1}}(u)\right) \leq n+2 n M^{\prime} .
$$

We deduce that $\Phi_{j+2} \mu_{i_{j+1}} \cdots \Phi_{2} \mu_{i_{1}} \Phi_{1}\left(x_{r}\right) \in B\left(m_{j+2}\right)$, where $m_{j+2}=n+2 n M^{\prime}$. Define $\gamma\left(X_{r}\right) \notin B\left(m_{k+1}\right)$.

When the recursion is complete, the extension of $\gamma$ to an element of $\mathcal{C}_{X}$ is not contained in $\mathcal{F}_{\sigma}$ for any $\sigma \in \Sigma$. This implies that $\gamma \notin\left\langle\mathcal{L}_{X}, \mu_{1}, \mu_{2}, \ldots\right\rangle$.

Next, we give three corollaries to the last result that demonstrate its potential applications. We start with a special case.

Corollary 2.2. Let $X=\mathbb{N}, \mathbb{Z}$ or $\mathbb{R}$ with the usual euclidean metric. Then $\operatorname{rank}\left(\mathcal{C}_{X^{n}}: \mathcal{L}_{X^{n}}\right)$ is uncountable for any $n \in \mathbb{N}$.

The next easy example, in conjunction with Corollary 2.2, demonstrates that although two different metrics on a space may induce the same topology, the conclusion of Theorem 2.1 can be true or false. In other words, Theorem 2.1] is dependent upon the metric used.

Example 2.3. Let $X=\mathbb{N}$ with metric $\rho(x, y)=1$ when $x \neq y$, and $\rho(x, y)=0$ when $x=y$. Then the topology induced by $\rho$ is the same as that induced by the usual euclidean metric. However, $\operatorname{rank}\left(\mathcal{C}_{X}: \mathcal{L}_{X}\right)=0$.

Next, we apply Theorem 2.1 in the context of endomorphisms of graphs. Recall that $\operatorname{End}_{X}(\Gamma)$ is the monoid of endomorphisms (i.e. mappings that preserve edges) of a graph $\Gamma$ with vertex set $X$ and $\mathcal{T}_{X}$ is the monoid of all mappings from $X$ to $X$.

Corollary 2.4. Let $\Gamma$ be a countably infinite connected graph with the vertex set $X$ with every vertex having finite degree. Then $\operatorname{rank}\left(\mathcal{T}_{X}: \operatorname{End}_{X}(\Gamma)\right)$ is uncountable.

Proof. Define a metric $d$ on $X$ by $d(x, y)=n$ if and only if the minimum length of a path from $x$ to $y$ is $n$. Of course, $d(x, y)=0$ if and only if $x=y$. Our metric satisfies the following conditions:

(i) for every $k \in \mathbb{N}$ there exist $x, y \in X$ such that $d(x, y)>k$;

(ii) for every $x \in X$ and every $k \in \mathbb{N}$ the set $\{y \in X: d(x, y) \leq k\}$ is finite.

Thus $(X, d)$ is a concentric space. Moreover, every mapping from $X$ to $X$ is continuous, and every endomorphism $\alpha$ of $\Gamma$ satisfies $d(\alpha(x), \alpha(y)) \leq d(x, y)$. In other words, $\mathcal{C}_{X}=\mathcal{T}_{X}$ and $\operatorname{End}_{X}(\Gamma) \subseteq \mathcal{L}_{X}$. Hence by Theorem 2.1] $\operatorname{rank}\left(\mathcal{T}_{X}: \operatorname{End}_{X}(\Gamma)\right)$ is uncountable.

Our next application of Theorem 2.1 concerns partially ordered sets. Let $X$ be a partially ordered set and let $\mathcal{O}_{X}$ denote the monoid of all order preserving mappings from $X$ to $X$. Recall, that for $x, y \in X$ a path from $x$ to $y$ of length $n$ is a sequence

$$
\left(x=p_{0}, p_{1}, \ldots, p_{n-1}, p_{n}=y\right)
$$

such that for each $i$ either $p_{i+1} \leq p_{i}$ or $p_{i+1} \geq p_{i}$. A partially ordered set is connected if there is a path between every pair of elements. For $x \in X$, the sets $\{y \in X: x \geq y\}$ and $\{y \in X: x \leq y\}$ are denoted by $x^{\vee}$ and $x^{\wedge}$, respectively. 
Corollary 2.5. Let $X$ be a connected partially ordered set with $\left|x^{\vee}\right|$ and $\left|x^{\wedge}\right|$ finite for every $x \in X$. Then $\operatorname{rank}\left(\mathcal{T}_{X}: \mathcal{O}_{X}\right)$ is uncountable.

Proof. For $x, y \in X$ define $d(x, y)$ to be the minimum length $n$ of a path from $x$ to $y$.

The remainder of the proof follows by the same argument as used in the proof of the previous corollary.

In fact, the converse of Corollary 2.5 is true; see the main theorem of 8 .

\section{Countable unions of COMPaCt Sets}

A topological space is called $\mathbf{K}_{\sigma}$ if it is a countable union of compact sets. Examples of such spaces are second countable locally compact spaces and all countable spaces. In particular, $\mathbb{Q}^{n}$ is $\mathbf{K}_{\sigma}, n \in \mathbb{N}$. In this section we prove an analogue of Theorem 2.1 for a class of $\mathbf{K}_{\sigma}$ spaces.

We require the following routine lemma.

Lemma 3.1. Let $\left(X, d_{1}\right),\left(Y, d_{2}\right)$ be metric spaces, let $\mu$ be a continuous map from $X$ to $Y$ and let $K$ be a compact subset of $X$. Then for each $\epsilon>0$ there exists $\delta>0$ such that for each $x \in K$ and any $y \in X$ with $d_{1}(x, y)<\delta$ we have $d_{2}(\mu(x), \mu(y))<\epsilon$.

Next, we give the main result of this section.

Theorem 3.2. Let $X$ be a $\mathbf{K}_{\sigma}$ metric space containing an extendible convergent sequence. Then $\operatorname{rank}\left(\mathcal{C}_{X}: \mathcal{L}_{X}\right)$ is uncountable.

Proof. We follow an argument similar, but not identical, to the proof of Theorem 2.1. As before we construct a continuous mapping $\gamma$ recursively that is not generated by $\mathcal{L}_{X}$ and a fixed countable set of mappings in $\mathcal{C}_{X}$.

Let $\left(x_{1}, x_{2}, \ldots\right)$ be an extendible convergent sequence in $X$ with limit $x$, let $K_{1} \subseteq K_{2} \subseteq \ldots$ be compact sets that comprise $X$, and let $\mu_{1}, \mu_{2}, \ldots \in \mathcal{C}_{X}$. Of course, we may assume that $x \notin\left\{x_{1}, x_{2}, \ldots\right\}$, that $x_{i} \neq x_{j}$ when $i \neq j$ and that $d\left(x_{i}, x\right) \leq 1 / i$. The set $\Sigma$ is defined in (11). For $[\vec{i}, n] \in \Sigma$ with $\vec{i}=\left(i_{1}, i_{2}, \ldots, i_{k}\right)$, $n \in \mathbb{N}$, the family of compositions $\mathcal{G}_{[\vec{i}, n]}$ is defined by

$$
\begin{aligned}
\mathcal{G}_{[\vec{i}, n]}=\left\{\Phi_{k+1} \mu_{i_{k}} \cdots \Phi_{2} \mu_{i_{1}} \Phi_{1}:\right. & \Phi_{j+1} \mu_{i_{j}} \cdots \Phi_{2} \mu_{i_{1}} \Phi_{1}(x) \in K_{n}, \\
& \left.\Phi_{j+1} \in \mathcal{L}_{X} \text { with constant } n, 0 \leq j \leq k\right\} .
\end{aligned}
$$

As before, enumerate the elements of $\Sigma$ as $\sigma_{1}, \sigma_{2}, \ldots$

Step 0 in the definition of $\gamma$ is to set $\gamma(x)=x$.

At step $r>0$ we will define $\gamma\left(x_{p_{r}}\right)=x_{r}$ for a point $x_{p_{r}}$ in such a way that when the recursion terminates $\gamma \notin \mathcal{G}_{\sigma_{r}}$. Moreover, this definition will ensure that $\left.\gamma\right|_{\left\{x, x_{1}, x_{2}, \ldots\right\}}$ is continuous when the recursion is complete. Assume that $\gamma$ has been defined on points $x_{p_{1}}, x_{p_{2}}, \ldots, x_{p_{r-1}}$ with images $x_{1}, x_{2}, \ldots, x_{r-1}$, respectively, and that $\sigma_{r}=[\vec{i}, n], \vec{i}=\left(i_{1}, \ldots, i_{k}\right)$ and $n \in \mathbb{N}$.

This step of the proof is completed by finding $x_{p_{r}}$, via a finite induction, such that every composition $\Phi_{k+1} \mu_{i_{k}} \cdots \Phi_{2} \mu_{i_{1}} \Phi_{1} \in \mathcal{G}_{\sigma_{r}}$ takes $x_{p_{r}}$ to a point closer to $x$ than $x_{r}$.

For the base case, by Lemma 3.1, if $\delta_{k+1}=d\left(x, x_{r}\right) / 2$, then there exists $\delta_{k}>0$ such that for $u \in K_{n}$ and $v \in X$ with $d(u, v)<\delta_{k}$ we have $d\left(\mu_{i_{k}}(u), \mu_{i_{k}}(v)\right)<$ $\delta_{k+1} / n$. Thus for such $u$ and $v, d\left(\Phi_{k+1} \mu_{i_{k}}(u), \Phi_{k+1} \mu_{i_{k}}(v)\right)<d\left(x, x_{r}\right) / 2$. 
The inductive hypothesis is as follows. For $0 \leq j \leq k-2$ we have chosen a sequence of positive numbers $\delta_{k+1}, \delta_{k}, \ldots, \delta_{k-j}$ such that if $0 \leq t \leq j, u \in K_{n}$ and $v \in X$ with $d(u, v)<\delta_{k-t}$, then

$$
d\left(\mu_{i_{k-t}}(u), \mu_{i_{k-t}}(v)\right)<\delta_{k-t+1} / n .
$$

For the inductive step, by Lemma 3.1, there exists $\delta_{k-j-1}>0$ such that for $u \in$ $K_{n}$ and $v \in X$ with $d(u, v)<\delta_{k-j-1}$ we have $d\left(\mu_{i_{k-j-1}}(u), \mu_{i_{k-j-1}}(v)\right)<\delta_{k-j} / n$.

When this finite induction is finished, we have a sequence $\delta_{k+1}, \delta_{k}, \ldots, \delta_{1}$ with the above properties. Let $p_{r}$ be chosen large enough to ensure that $d\left(x, x_{p_{r}}\right) \leq \delta_{1} / n$ and that $x_{p_{r}} \notin\left\{x_{p_{1}}, \ldots, x_{p_{r-1}}\right\}$. It follows that

$$
\begin{aligned}
& d\left(\Phi_{k+1} \mu_{i_{k}} \cdots \Phi_{2} \mu_{i_{1}} \Phi_{1}(x), \Phi_{k+1} \mu_{i_{k}} \cdots \Phi_{2} \mu_{i_{1}} \Phi_{1}\left(x_{p_{r}}\right)\right) \\
& \quad \leq \delta_{k+1}=d\left(x, x_{r}\right) / 2<d\left(x, x_{r}\right) .
\end{aligned}
$$

So, if we define $\gamma\left(x_{p_{r}}\right)=x_{r}$, then no element of $\mathcal{C}_{X}$ that agrees with $\gamma$ on the points $x_{p_{1}}, \ldots, x_{p_{r}}$ is an element of $\mathcal{G}_{\sigma_{r}}$.

When the recursion is finished and $\gamma$ is already defined on every element of $\left\{x_{p_{1}}, x_{p_{2}}, \ldots\right\} \cup\{x\}$, we extend $\gamma$ to the whole set $\left\{x_{1}, x_{2}, \ldots\right\} \cup\{x\}$ by defining $\gamma\left(x_{s}\right)=x$ for all $s \notin\left\{p_{1}, p_{2}, \ldots\right\}$. As the sequence $x_{n}$ is convergent to $x$ the function $\gamma$, as defined so far, on the elements $x, x_{1}, x_{2} \ldots$, is continuous. Thus $\gamma$ can be extended to an element of $\mathcal{C}_{X}$, and, by construction, this element is not a member of any $\mathcal{G}_{\sigma_{r}}$.

In the next section we give examples that demonstrate the necessity of every assumption in Theorem 3.2 and Corollary 3.3 .

Corollary 3.3. Let $X$ be a locally compact separable metric space containing an extendible convergent sequence. Then $\operatorname{rank}\left(\mathcal{C}_{X}: \mathcal{L}_{X}\right)$ is uncountable.

Proof. Every locally compact separable metric space is a $\mathbf{K}_{\sigma}$ metric space.

Corollary 3.4. Let $X$ be a 0 -dimensional $\mathbf{K}_{\sigma}$ metric space that contains a convergent sequence with an infinite number of distinct terms. Then $\operatorname{rank}\left(\mathcal{C}_{X}: \mathcal{L}_{X}\right)$ is uncountable.

Proof. Assume that $\left(x_{1}, x_{2}, \ldots\right)$ is a convergent sequence with limit $x$, that $x_{i} \neq x_{j}$ if $i \neq j$ and $x \notin\left\{x_{1}, x_{2}, \ldots\right\}$. It is enough to show that this sequence is extendible.

As $X$ is 0 -dimensional and metric, we can take pairwise disjoint clopen neighbourhoods $H_{i}$ of $x_{i}, i \in \mathbb{N}$, none of which contains $x$ and such that $\operatorname{diam}\left(H_{i}\right) \rightarrow 0$, with $i \rightarrow \infty$. Let $\mu$ be any continuous mapping from $\left\{x_{n}: n \in \mathbb{N}\right\} \cup\{x\}$ to $\left\{x_{n}: n \in \mathbb{N}\right\} \cup\{x\}$. Define $\mu^{*}: X \rightarrow X$ by

$$
\mu^{*}(u)= \begin{cases}\mu\left(x_{i}\right), & u \in H_{i}, \\ \mu(x), & \text { otherwise }\end{cases}
$$

Obviously, $\mu^{*} \in \mathcal{C}_{X}$.

As every countable metric space is a 0 -dimensional $\mathbf{K}_{\sigma}$ space, we have the following corollary.

Corollary 3.5. Let $X$ be a countable metric space that contains a convergent sequence with infinitely many distinct terms (i.e. $X$ contains at least one accumulation point). Then $\operatorname{rank}\left(\mathcal{C}_{X}: \mathcal{L}_{X}\right)$ is uncountable. 


\section{Counterexamples}

In this section we give two examples. Namely, an example that the extendible sequence assumption in Theorem 3.2 may not be abandoned, and a further example that Corollary 3.3 does not hold when $X$ is locally compact but not separable. Another example that demonstrates that Theorem 3.2 does not hold when $X$ has an extendible sequence but is not $\mathbf{K}_{\sigma}$ is given in the next section. Our first example comes from the literature.

Example 4.1. In [2] it is proven that there exist metric continua (i.e. compact and connected metric spaces) with no continuous mappings other than the constant mappings and the identity. It follows that every continuous mapping on such a continua $\mathfrak{C}$ is Lipschitz, and so $\operatorname{rank}\left(\mathcal{C}_{\mathfrak{C}}: \mathcal{L}_{\mathfrak{C}}\right)=0$. Such a space is clearly $\mathbf{K}_{\sigma}$ but contains no extendible convergent sequences. Continua with this property can even be planar; see [11].

Our next example demonstrates that the separability assumption in Corollary 3.3 cannot, in general, be removed.

Example 4.2. Let $X$ be a direct sum of continuum copies of the unit interval $[0,1]$ with the usual euclidean metric. In other words, $X$ is the product space

$$
X=[0,1] \times \mathfrak{c},
$$

where $\mathfrak{c}$ denotes the cardinal number continuum with the discrete topology. We prove that there exists a mapping $\Psi$ such that $\left\langle\mathcal{L}_{X}, \Psi\right\rangle=\mathcal{C}_{X}$. Define a metric $d$ on $X$ by

$$
d((s, \alpha),(t, \beta))= \begin{cases}|s-t|, & \alpha=\beta, \\ 1, & \alpha \neq \beta .\end{cases}
$$

The metric $d$ is consistent with the topology of $X$. Clearly, $X$ is locally compact but not separable. Note that a continuous mapping $\mu: X \rightarrow X$ maps each component $[0,1] \times\{\alpha\}$ into some other component $[0,1] \times\{\beta\}$. Thus componentwise $\mu$ is a continuous function from $[0,1]$ into $[0,1]$. There are continuum many members of $\mathcal{C}_{[0,1]}$. Let $\mathcal{C}_{[0,1]}=\left\{\mu_{\alpha}: \alpha<\mathfrak{c}\right\}$, and let $\tau: \mathfrak{c} \times \mathfrak{c} \rightarrow \mathfrak{c}$ be a bijection.

We define the mapping $\Psi \in \mathcal{C}_{X}$ by

$$
\Psi(t, \tau(\alpha, \beta))=\left(\mu_{\alpha}(t), \beta\right) .
$$

Let $\mu \in \mathcal{C}_{X}$ be arbitrary. By what we said above, there are two mappings $\xi: \mathfrak{c} \rightarrow \mathfrak{c}$ and $\zeta: \mathfrak{c} \rightarrow \mathfrak{c}$ such that

$$
\mu(t, \alpha)=\left(\mu_{\xi(\alpha)}(t), \zeta(\alpha)\right)
$$

for each $t \in[0,1]$ and $\alpha \in \mathfrak{c}$. Let $\Phi: X \rightarrow X$ be defined as

$$
\Phi(t, \alpha)=(t, \tau(\xi(\alpha), \zeta(\alpha)))
$$

It is easy to see that $\Phi \in \mathcal{L}_{X}$. Now we have

$$
\mu(t, \alpha)=\left(\mu_{\xi(\alpha)}(t), \zeta(\alpha)\right)=\Psi(t, \tau(\xi(\alpha), \zeta(\alpha)))=\Psi \circ \Phi(t, \alpha) .
$$

Thus $\mu$ is a composition of $\Phi$ and $\Psi$, and $\operatorname{son} \operatorname{rank}\left(\mathcal{C}_{X}: \mathcal{L}_{X}\right)=1$. 


\section{A FURTHER COUNTEREXAMPLE}

In this section we give a further example. This example takes the form of a theorem and shows that Theorem 3.2 does not necessarily hold when $X$ is a (separable) metric space that has an extendible sequence but is not $\mathbf{K}_{\sigma}$. The space we consider is closely related to a classical space that plays a crucial role in descriptive set theory, namely, the Baire space $\mathbb{N}^{\mathbb{N}}=\mathcal{N}$. Recall, that for $x=\left(x_{i}\right)_{i \in \mathbb{N}}, y=\left(y_{i}\right)_{i \in \mathbb{N}} \in \mathcal{N}$ the distance $d(x, y)=1 / n$, where $n$ is the least number where $x_{n} \neq y_{n}$. For clarity, we may write the $n^{\text {th }}$ element $x_{n}$ of a sequence $x$ as $x(n)$. Of course, $\mathcal{N}$ contains an extendible convergent sequence but is not $\mathbf{K}_{\sigma}$.

The space that we wish to consider is just $\mathcal{N}$ with a single point removed, the sequence $(1,1, \ldots)$; we denote this space by $\mathcal{N}^{*}$. The new space $\mathcal{N}^{*}$ is separable and contains an extendible convergent sequence but is not $\mathbf{K}_{\sigma}$ (and hence not locally compact). The reader may ask why we consider $\mathcal{N}^{*}$ rather than $\mathcal{N}$. This question is answered by the main result in the next section, where we prove that $\operatorname{rank}\left(\mathcal{C}_{\mathcal{N}}: \mathcal{L}_{\mathcal{N}}\right)=\aleph_{1}$. However, removing a single point from $\mathcal{N}$ we obtain the following result, which is the main theorem of this section.

Theorem 5.1. The relative rank of $\mathcal{C}_{\mathcal{N}^{*}}$ modulo $\mathcal{L}_{\mathcal{N}^{*}}$ is 1 .

Before giving the proof of this theorem, we require some notation and additional lemmas. We now define a metric $\rho$ on the product space $\mathcal{N}^{\mathbb{N}}$. First, define $\kappa: \mathbb{N}^{2} \rightarrow$ $\mathbb{N}$ by

$$
\kappa(i, j)=\frac{(i+j-1)(i+j-2)}{2}+j .
$$

This function corresponds to enumerating $\mathbb{N}^{2}$ as follows:

$$
(1,1),(2,1),(1,2),(3,1),(2,2),(1,3), \ldots
$$

Thus $\kappa$ is a bijection. Note that

$$
\text { if } p+q<s+t \text {, then } \kappa(p, q)<\kappa(s, t),
$$

and $\kappa(p, q) \geq p, q$. Let $m$ and $n$ be the coordinate functions of $\kappa^{-1}$, i.e. functions such that $\kappa^{-1}(i)=(m(i), n(i))$. Let $x=\left(x_{1}, x_{2}, \ldots\right)$ and define $\varphi: \mathcal{N} \rightarrow \mathcal{N}^{\mathbb{N}}$ by

$$
\varphi(x)=\left(y^{(1)}, y^{(2)}, \ldots\right) \text { where } y_{q}^{(p)}=x_{\kappa(p, q)},
$$

(i.e. $\left.x_{k}=x_{\kappa(m(k), n(k))}=y_{n(k)}^{(m(k))}\right)$. Now, for $u=\left(u^{(1)}, u^{(2)}, \ldots\right), v=\left(v^{(1)}, v^{(2)}, \ldots\right)$ $\in \mathcal{N}^{\mathbb{N}}$, define

$$
\rho(u, v)=d\left(\varphi^{-1}(u), \varphi^{-1}(v)\right)=d\left(\left(u_{n(1)}^{m(1)}, u_{n(2)}^{m(2)}, \ldots\right),\left(v_{n(1)}^{m(1)}, v_{n(2)}^{m(2)}, \ldots\right)\right)=\frac{1}{i},
$$

where $i=\min \left\{j \in \mathbb{N}: u_{n(j)}^{(m(j))} \neq v_{n(j)}^{(m(j))}\right\}$.

In other words, the metric $\rho$ is the metric $d$ transported from the space $\mathcal{N}$ to the space $\mathcal{N}^{\mathbb{N}}$. The definition of the metric $\rho$ is contrived to make $\varphi$ and its inverse $\varphi^{-1}$ Lipschitz homeomorphisms with constant 1. From now on, unless specified otherwise, we always treat $\mathcal{N}^{\mathbb{N}}$ and its subsets as metric spaces equipped with the metric $\rho$. It is known that the product topology on $\mathcal{N}^{\mathbb{N}}$ is generated by the metric

$$
\bar{\rho}(x, y)=\sum_{i=1}^{\infty} \frac{1}{2^{i}} d\left(x^{(i)}, y^{(i)}\right)
$$


where $x=\left(x^{(1)}, x^{(2)}, \ldots\right)$ and $y=\left(y^{(1)}, y^{(2)}, \ldots\right)$. As the topology on $\left(\mathcal{N}^{\mathbb{N}}, \rho\right)$ is given as the image by $\varphi$ of the topology on $\mathcal{N}$, we infer that the topologies on $\mathcal{N}^{\mathbb{N}}$ generated by $\bar{\rho}$ and $\rho$ are identical.

Lemma 5.2. Let $A \subseteq \mathcal{N}$ and let $\zeta_{i}: A \rightarrow \mathcal{N}$ be Lipschitz mappings with constant $1, i \in \mathbb{N}$. Then the mapping $\phi: A^{\mathbb{N}} \rightarrow \mathcal{N}^{\mathbb{N}}$ defined by $\phi\left(x^{(1)}, x^{(2)}, \ldots\right)=$ $\left(\zeta_{1}\left(x^{(1)}\right), \zeta_{2}\left(x^{(2)}\right), \ldots\right)$ is Lipschitz with constant 1 .

Proof. Let $x=\left(x^{(1)}, x^{(2)}, \ldots\right), y=\left(y^{(1)}, y^{(2)}, \ldots\right) \in A^{\mathbb{N}}$ such that $\rho(x, y)=1 / p$, for some $p$. From (5), we have that $p=\min \left\{i: x_{n(i)}^{(m(i))} \neq y_{n(i)}^{(m(i))}\right\}$. Thus for any $j<p, x_{n(j)}^{(m(j))}=y_{n(j)}^{(m(j))}$. Moreover, if $r<n(j)$, then $r+m(j)<n(j)+$ $m(j)$, and so $\kappa(m(j), r)<\kappa(m(j), n(j))=j$ by (3). It follows that $x_{r}^{(m(j))}=$ $y_{r}^{(m(j))}$, and so $d\left(x^{(m(j))}, y^{(m(j))}\right)<1 / n(j)$. Since $\zeta_{m(j)}$ is Lipschitz with constant $1, d\left(\zeta_{m(j)}\left(x^{(m(j))}\right), \zeta_{m(j)}\left(y^{(m(j))}\right)\right) \leq d\left(x^{(m(j))}, y^{(m(j))}\right)<1 / n(j)$.

So, if $\zeta_{m(j)}\left(x^{(m(j))}\right)=u^{(m(j))}$ and $\zeta_{m(j)}\left(y^{(m(j))}\right)=v^{(m(j))}$, then $u_{n(j)}^{(m(j))}=v_{n(j)}^{(m(j))}$ for all $j<p$. But $d(\phi(x), \phi(y))=1 / q$ where $q=\min \left\{i: u_{n(i)}^{(m(i))} \neq v_{n(i)}^{(m(i))}\right\}$, and so $d(\phi(x), \phi(y))<1 / p=d(x, y)$.

For $\vec{\phi} \in \mathbb{N}<\mathbb{N}$ denote by $[\vec{\phi}]$ the clopen set of all elements in $\mathcal{N}$ that agree with $\vec{\phi}$ on the first $|\vec{\phi}|$ components. For $\vec{\phi} \in \mathbb{N}<\mathbb{N}$ and $\vec{\theta} \in \mathbb{N}<\mathbb{N}$ or $\theta \in \mathcal{N}$ denote by $\vec{\phi} \wedge \vec{\theta}$ or $\vec{\phi} \wedge \theta$ the element of $\mathbb{N}<\mathbb{N}$ or $\mathcal{N}$, respectively, formed by concatenating the sequences $\vec{\phi}$ and $\vec{\theta}$ or $\theta$. For $\vec{\phi} \in \mathbb{N}<\mathbb{N}$ denote by $\vec{\phi}^{[i]}, \vec{\phi}$ concatenated with itself $i$ times; if $i=0$, then suppose that $\vec{\phi}^{[i]}$ is empty.

Lemma 5.3. There is a homeomorphism $\eta$ from $\left(\mathcal{N}^{*}\right)^{\mathbb{N}}$ to $\mathcal{N}^{*}$ that is Lipschitz with constant 1 .

Proof. We define $\eta$ by defining a sequence of homeomorphisms $\phi:\left(\mathcal{N}^{*}\right)^{\mathbb{N}} \rightarrow \mathcal{N}^{\mathbb{N}}$, $\psi: \mathcal{N}^{\mathbb{N}} \rightarrow \mathcal{N}$ and $\theta: \mathcal{N} \rightarrow \mathcal{N}^{*}$, each of which is Lipschitz with constant 1 .

Before making the definition of $\phi$, define $\zeta: \mathcal{N}^{*} \rightarrow \mathcal{N}$ by

$$
\zeta(x)= \begin{cases}x & \text { if } x=\left(1, a_{2}, \ldots\right), a_{2}>1, \\ (1)^{[k]} \wedge\left(2 a_{k+1}-2, a_{k+2}, \ldots\right) & \text { if } x=(1)^{[k]} \wedge\left(a_{k+1}, a_{k+2}, \ldots\right), \\ & a_{k+1}>1, k>1, \\ (1)^{\left[a_{2}+1\right]} \wedge\left(2 a_{3}+1, a_{4}, \ldots\right) & \text { if } x=\left(2, a_{2}, a_{3}, \ldots\right), \\ \left(a_{1}-1, a_{2}, \ldots\right) & \text { if } x=\left(a_{1}, a_{2}, \ldots\right), a_{1}>2 .\end{cases}
$$

It may be verified that this definition of $\zeta$ gives a homeomorphism that is Lipschitz with constant 1 from $\mathcal{N}^{*}$ to $\mathcal{N}$. Note that $\zeta^{-1}$ is not Lipschitz. The mapping $\phi:\left(\mathcal{N}^{*}\right)^{\mathbb{N}} \rightarrow \mathcal{N}^{\mathbb{N}}$ defined by applying $\zeta$ componentwise is a homeomorphism that is Lipschitz with constant 1 by Lemma 5.2 .

The mapping $\psi$ is defined by $\psi=\varphi^{-1}: \mathcal{N}^{\mathbb{N}} \rightarrow \mathcal{N}$, where $\varphi$ is defined by (4), and is a Lipschitz homeomorphism with constant 1.

Penultimately, define $\theta: \mathcal{N} \rightarrow \mathcal{N}^{*}$ by

$$
\theta(x)= \begin{cases}\left(i+1, a_{2}, a_{3}, \ldots\right) & \text { if } x=\left(2 i-1, a_{2}, a_{3}, \ldots\right), i \geq 1 \\ (1)^{[i]} \wedge\left(a_{2}+1, a_{3}, \ldots\right) & \text { if } x=\left(2 i, a_{2}, a_{3}, \ldots\right), i \geq 1\end{cases}
$$

Again, it may be verified that this definition gives a homeomorphism which is Lipschitz with constant 1. 
Ultimately, the composition $\eta=\theta \circ \psi \circ \phi:\left(\mathcal{N}^{*}\right)^{\mathbb{N}} \rightarrow \mathcal{N}^{*}$ is a homeomorphism that is Lipschitz with constant 1.

A subset $A$ of a topological space $X$ is discrete if for each point $x$ in $A$ there is an open set $V$ in $X$ such that $V \cap A=\{x\}$. We will call a continuous mapping $\mu$ from $A$ to $B$ discrete if $\mu(A)$ is discrete.

Lemma 5.4. Let $S$ be a dense subset of $\mathcal{N}^{*}$ and let $\nu: \mathcal{N}^{*} \rightarrow \mathcal{N}$ be a continuous mapping. Then there exist continuous discrete $\nu_{1}, \nu_{2}, \ldots: \mathcal{N}^{*} \rightarrow S$ such that

for all $i, j \geq n$ and $x \in \mathcal{N}^{*}, d\left(\nu_{i}(x), \nu_{j}(x)\right) \leq 1 / n$ and $\nu_{i} \rightarrow \nu$ pointwise as $i \rightarrow \infty$.

Proof. Let $i \in \mathbb{N}$ be arbitrary. The clopen sets $[\vec{\gamma}]$, for $\vec{\gamma} \in \mathbb{N}<\mathbb{N}$ and $|\vec{\gamma}|=i$, partition $\mathcal{N}$. Thus the sets $\nu^{-1}[\vec{\gamma}]$ partition $\mathcal{N}^{*}$. Define $\nu_{i}(x)=s_{\vec{\gamma}}$ for all $x \in \nu^{-1}[\vec{\gamma}]$, where $s_{\vec{\gamma}} \in[\vec{\gamma}] \cap S$ is fixed for each $\vec{\gamma} \in \mathbb{N}^{<\mathbb{N}}$. Thus each $\nu_{i}$, being constant on each of the sets $\nu^{-1}[\vec{\gamma}]$, is continuous. Moreover, the sequence $\nu_{1}, \nu_{2}, \ldots$ has the required property.

Lemma 5.5. Let $S$ be a countable dense subset of $\mathcal{N}^{*}$. Then there exists a continuous mapping $\Psi: \mathcal{N}^{*} \rightarrow \mathcal{N}^{*}$ such that for every continuous discrete mapping $\mu: \mathcal{N}^{*} \rightarrow S$ there is a Lipschitz mapping $\Phi_{\mu}: \mathcal{N}^{*} \rightarrow \mathcal{N}^{*}$ with constant 1 such that $\mu=\Psi \circ \Phi_{\mu}$.

Proof. Take any sequence $\sigma=\left(s_{1}, s_{2}, \ldots\right)$ of elements of $S$ such that every element of $S$ occurs infinitely many times in $\sigma$. We define the function $\Psi$ in two stages. First, for every $n \in \mathbb{N}$ and $x \in\left[(1)^{[n]} \wedge(2)\right]$ define $\Psi$ by

$$
\Psi(x)=s_{n} .
$$

Note that the set $\bigcup_{n \in \mathbb{N}}\left[(1)^{[n]} \wedge(2)\right]$, on which $\Psi$ was defined at the first stage, is a clopen set in $\mathcal{N}^{*}$. It follows that the definition of $\Psi$ can be completed so that $\Psi$ is continuous on $\mathcal{N}^{*}$. This can be done, for instance, by setting $\Psi$ to be constant on the remaining part of $\mathcal{N}^{*}$.

Let $\mu: \mathcal{N}^{*} \rightarrow S$ be any discrete continuous mapping. We partition the preimage of each $s \in \mu\left(\mathcal{N}^{*}\right)$ into open sets $\left[\vec{\sigma}_{s, k}\right]$ for natural numbers $k$ and $\vec{\sigma}_{s, k} \in \mathbb{N}<\mathbb{N}$. Now, we may define $\Phi_{\mu}$ on $x=\left(x_{1}, x_{2}, \ldots\right) \in\left[\vec{\sigma}_{s, k}\right]$ by

$$
\Phi_{\mu}(x)=(1)^{\left[N\left(\vec{\sigma}_{s, k}\right)\right]} \wedge\left(2, x_{m+1}, x_{m+2}, \ldots\right),
$$

where $m=\left|\vec{\sigma}_{s, k}\right|$ and $N\left(\vec{\sigma}_{s, k}\right)$ is the least number greater than $m$ such that $s=$ $s_{N\left(\vec{\sigma}_{s, k}\right)}$.

In order to prove that $\Phi_{\mu}$ is a Lipschitz mapping with constant 1 , let $x, y \in \vec{\sigma}_{s, k}$, $x \neq y$, for some $s \in \mu\left(\mathcal{N}^{*}\right)$ and $k \in \mathbb{N}$. Again let $m=\left|\vec{\sigma}_{k, s}\right|$ and let $d(x, y)=1 / p$, where $p=m+\min \left\{i: x_{m+i} \neq y_{m+i}\right\}$. Then

$$
d\left(\Phi_{\mu}(x), \Phi_{\mu}(y)\right)=1 /\left(N\left(\vec{\sigma}_{s, k}\right)+1+p-m\right) .
$$

Since $N\left(\vec{\sigma}_{s, k}\right) \geq m$ we have $d\left(\Phi_{\mu}(x), \Phi_{\mu}(y)\right) \leq d(x, y)$.

The other case is that $x \in\left[\vec{\sigma}_{s, k}\right]$ and $y \in\left[\vec{\sigma}_{t, l}\right]$ with $\left[\vec{\sigma}_{s, k}\right] \neq\left[\vec{\sigma}_{t, l}\right]$ (in which case $\left.\left[\vec{\sigma}_{s, k}\right] \cap\left[\vec{\sigma}_{t, l}\right]=\emptyset\right)$. Now, if $M=\min \left\{\left|\vec{\sigma}_{s, k}\right|,\left|\vec{\sigma}_{t, l}\right|\right\}$, then $d(x, y) \geq 1 / M$. The images $\Phi_{\mu}(x)$ and $\Phi_{\mu}(y)$ agree on at least the first $P=\min \left\{N\left(\left|\vec{\sigma}_{s, k}\right|\right), N\left(\left|\vec{\sigma}_{t, l}\right|\right)\right\}$ terms. Therefore $d\left(\Phi_{\mu}(x), \Phi_{\mu}(y)\right) \leq 1 / P$. But $P \geq M$, and so $d\left(\Phi_{\mu}(x), \Phi_{\mu}(y)\right) \leq d(x, y)$.

To complete the proof we must show that $\mu=\Psi \circ \Phi_{\mu}$. Let $x \in \mathcal{N}^{*}$ be arbitrary. 
Then there exist $k, s$ such that $x \in\left[\vec{\sigma}_{k, s}\right] \subseteq \mu^{-1}(s)$. If $m=\left|\vec{\sigma}_{k, s}\right|$, then

$$
\Psi \circ \Phi_{\mu}(x)=\Psi\left((1)^{\left[N\left(\vec{\sigma}_{k, s}\right)\right]} \wedge\left(2, x_{m+1}, x_{m+2}, \ldots\right)\right)=s_{N\left(\vec{\sigma}_{k, s}\right)}=s=\mu(x),
$$

and the proof is complete.

Lemma 5.6. The mapping $\tau: \mathcal{N} \rightarrow \mathcal{N}^{\mathbb{N}}$ defined by $\tau(x)=(x, x, \ldots)$ is Lipschitz with constant 1.

Proof. Let $x, y \in \mathcal{N}, x \neq y$, and let $\varphi: \mathcal{N} \rightarrow \mathcal{N}^{\mathbb{N}}$ be the mapping defined by (4). Then $\varphi^{-1}(\tau(x))=\varphi^{-1}(x, x, \ldots)=\left(x_{n(1)}, x_{n(2)}, \ldots\right)$ and, likewise, $\varphi^{-1}(\tau(y))=$ $\left(y_{n(1)}, y_{n(2)}, \ldots\right)$. Hence

$$
\rho(\tau(x), \tau(y))=d\left(\left(x_{n(1)}, x_{n(2)}, \ldots\right),\left(y_{n(1)}, y_{n(2)}, \ldots\right)\right)=\frac{1}{i},
$$

where $i=\min \left\{j: x_{n(j)} \neq y_{n(j)}\right\}$. In particular, $x_{n(i)} \neq y_{n(i)}$, and so $d(x, y) \geq$ $1 / n(i)$. But $i=\kappa(m(i), n(i)) \geq n(i)$, and so $d(x, y) \geq 1 / i=\rho(\tau(x), \tau(y))$.

We may now commence with the proof of the main theorem of this section.

Proof of Theorem [5.1, Let $\zeta: \mathcal{N}^{*} \rightarrow \mathcal{N}$ and $\eta:\left(\mathcal{N}^{*}\right)^{\mathbb{N}} \rightarrow \mathcal{N}^{*}$ be homeomorphisms, such as those defined in (77) and Lemma 5.3, and let $\Psi: \mathcal{N}^{*} \rightarrow \mathcal{N}^{*}$ be the mapping defined in Lemma 5.5 for a fixed countable dense subset $S$ of $\mathcal{N}^{*}$ (and hence $\mathcal{N}$ ).

Let $\mathcal{Z}$ denote the set of all elements $\left(z^{(i)}\right)_{i \in \mathbb{N}}$ of $\left(\mathcal{N}^{*}\right)^{\mathbb{N}}$ satisfying

$$
d\left(z^{(i)}, z^{(j)}\right) \leq 1 / k \text { whenever } i, j \geq k .
$$

The set $\mathcal{Z}$ is closed in $\left(\mathcal{N}^{*}\right)^{\mathbb{N}}$ (this is easy to see when one considers $\mathcal{N}^{\mathbb{N}}$ with the metric $\bar{\rho}$ defined by (6) $)$. Therefore, by [4], $\mathcal{Z}$ is a retract of $\left(\mathcal{N}^{*}\right)^{\mathbb{N}}$. Denote by $\sigma$ the retraction from $\left(\mathcal{N}^{*}\right)^{\mathbb{N}}$ to $\mathcal{Z}$.

Define $\Psi^{\prime}:\left(\mathcal{N}^{*}\right)^{\mathbb{N}} \rightarrow\left(\mathcal{N}^{*}\right)^{\mathbb{N}}$ by

$$
\Psi^{\prime}\left(x^{(1)}, x^{(2)}, \ldots\right)=\left(\Psi\left(x^{(1)}\right), \Psi\left(x^{(2)}\right), \ldots\right)
$$

Of course, $\Psi^{\prime}$ is continuous since $\Psi$ is. If $y \in \mathcal{N}^{*}$, then $\eta^{-1}(y) \in\left(\mathcal{N}^{*}\right)^{\mathbb{N}}$. Therefore $\sigma \circ \Psi^{\prime} \circ \eta^{-1}(y)$ is an element $\left(z^{(1)}, z^{(2)}, \ldots\right)$ of $\mathcal{Z}$. The mapping $\lambda: \mathcal{Z} \rightarrow \mathcal{N}$ defined by

$$
\lambda\left(z^{(1)}, z^{(2)}, \ldots\right)=\lim _{i \rightarrow \infty} z^{(i)} \in \mathcal{N}
$$

is well defined and continuous. Define $\Lambda \in \mathcal{C}_{\mathcal{N}^{*}}$ by

$$
\Lambda(y)=\zeta^{-1} \circ \lambda \circ \sigma \circ \Psi^{\prime} \circ \eta^{-1}(y) .
$$

It is this mapping $\Lambda$ that will, together with the Lipschitz mappings, allow us to generate every continuous mapping from $\mathcal{N}^{*}$ to itself.

In order to prove this, let $\mu \in \mathcal{C}_{\mathcal{N}^{*}}$ be arbitrary. We will write $\mu$ as a composition of a single element of $\mathcal{L}_{\mathcal{N}^{*}}$ and $\Lambda$. For the time being consider the continuous mapping $\nu=\zeta \circ \mu: \mathcal{N}^{*} \rightarrow \mathcal{N}$. By Lemma 5.4 there exist continuous discrete $\nu_{1}, \nu_{2}, \ldots: \mathcal{N}^{*} \rightarrow S$ that satisfy condition (8). Thus, for $x \in \mathcal{N}^{*}$ the sequence $\left(\nu_{1}(x), \nu_{2}(x), \ldots\right)$ is an element of $\mathcal{Z}$. By Lemma 5.5, each of the mappings $\nu_{i}$ can be written as a composition $\Psi \circ \Phi_{\nu_{i}}$ for some $\Phi_{\nu_{i}} \in \mathcal{L}_{\mathcal{N}^{*}}$ with constant 1 . Now, by Lemmas 5.6 and 5.2 the mapping $\alpha$ that takes $x \in \mathcal{N}^{*}$ to $\left(\Phi_{\nu_{1}}(x), \Phi_{\nu_{2}}(x), \ldots\right) \in$ $\left(\mathcal{N}^{*}\right)^{\mathbb{N}}$ is continuous and Lipschitz. Therefore the mapping $\Phi: \mathcal{N}^{*} \rightarrow \mathcal{N}^{*}$ defined by

is continuous and Lipschitz.

$$
\Phi(x)=\eta \circ \alpha(x)
$$


The proof is concluded by observing that for $x \in \mathcal{N}^{*}$ we have

$$
\begin{aligned}
\Lambda \circ \Phi(x) & =\zeta^{-1} \circ \lambda \circ \sigma \circ \Psi^{\prime} \circ \eta^{-1} \circ \eta \circ \alpha(x)=\zeta^{-1} \circ \lambda \circ \sigma \circ \Psi^{\prime}\left(\Phi_{\nu_{1}}(x), \Phi_{\nu_{2}}(x), \ldots\right) \\
& =\zeta^{-1} \circ \lambda \circ \sigma\left(\Psi \circ \Phi_{\nu_{1}}(x), \Psi \circ \Phi_{\nu_{2}}(x), \ldots\right)=\zeta^{-1} \circ \lambda \circ \sigma\left(\nu_{1}(x), \nu_{2}(x), \ldots\right) \\
& =\zeta^{-1} \circ \lambda\left(\nu_{1}(x), \nu_{2}(x), \ldots\right)=\zeta^{-1}\left(\lim _{i \rightarrow \infty} \nu_{i}(x)\right)=\zeta^{-1} \circ \nu(x) \\
& =\zeta^{-1} \circ \zeta \circ \mu(x)=\mu(x) .
\end{aligned}
$$

In other words, $\mu \in\left\langle\mathcal{L}_{\mathcal{N}^{*}}, \Lambda\right\rangle$, and so $\left\langle\mathcal{L}_{\mathcal{N}^{*}}, \Lambda\right\rangle=\mathcal{C}_{\mathcal{N}^{*}}$.

\section{The BAIRE SPACE}

It is well known that $\mathcal{N}$ is homeomorphic to $\mathcal{N}^{2}$ and that such a homeomorphism may be chosen to be Lipschitz in both directions. For example, the mapping $\sigma\left(x_{1}, x_{2}, x_{3}, x_{4}, \ldots\right)=\left(\left(x_{1}, x_{3}, \ldots\right),\left(x_{2}, x_{4}, \ldots\right)\right)$ is such a Lipschitz homeomorphism. The space $\mathcal{N}$ is homeomorphic to the irrationals with the usual euclidean topology [10, Section 3, IX].

We now state the main result of this section.

Theorem 6.1. The relative rank of $\mathcal{C}_{\mathcal{N}}$ modulo $\mathcal{L}_{\mathcal{N}}$ is $\aleph_{1}$.

The proof of this theorem is divided into two parts, the first being a proof that $\operatorname{rank}\left(\mathcal{C}_{\mathcal{N}}: \mathcal{L}_{\mathcal{N}}\right) \geq \aleph_{1}$ and another that $\operatorname{rank}\left(\mathcal{C}_{\mathcal{N}}: \mathcal{L}_{\mathcal{N}}\right) \leq \aleph_{1}$.

Now, we begin a sequence of results leading to the first of these proofs. Let $\mathcal{A} \subseteq \mathcal{T}_{\mathcal{N}}$ and $X \subseteq \mathcal{N} \times \mathcal{N}$. A mapping $\Psi: X \rightarrow \mathcal{N}$ is called universal for $\mathcal{A}$ if $\mathcal{A} \subseteq\{\Psi(x, \cdot): x \in \mathcal{N}\}$.

The following proposition is well known, however, for completeness a proof is included.

Proposition 6.2. There is no continuous mapping from $\mathcal{N} \times \mathcal{N}$ to $\mathcal{N}$ that is universal for $\mathcal{C}_{\mathcal{N}}$.

Proof. Assume that there is such a mapping, call it $\Gamma$. Then the mapping $\gamma(x)=$ $\Gamma(x, x)$ is continuous. Now, for all $\xi \in \mathcal{C}_{\mathcal{N}}$ there exists $x \in \mathcal{N}$ such that $\gamma(x)=\xi(x)$. But if $\gamma_{1}, \gamma_{2}, \ldots$ are the coordinate functions of $\gamma$, then the continuous mapping $\xi: \mathcal{N} \rightarrow \mathcal{N}$ defined by $\xi(x)=\left(\gamma_{1}(x)+1, \gamma_{2}(x), \ldots\right) \in \mathcal{C}_{\mathcal{N}}$ shares no common value with $\gamma$, a contradiction.

However, there are continuous mappings from some proper subsets of $\mathcal{N} \times \mathcal{N}$ to $\mathcal{N}$ that are universal for $\mathcal{C}_{\mathcal{N}}$. We use this fact later; see Lemma 6.5.

Lemma 6.3. There exists a continuous mapping $\Lambda: \mathcal{N} \times \mathcal{N} \rightarrow \mathcal{N}$ universal for $\mathcal{L}_{\mathcal{N}}$.

Proof. Let $\tau_{k}: \mathbb{N}^{k-1} \rightarrow \mathbb{N}$ be a bijection. For a sequence $x=\left(x_{1}, x_{2}, \ldots\right) \in \mathcal{N}$ let $x(i)=x_{i}$ and let $\mathcal{L}_{\mathcal{N}}^{(k)}$ denote the class of Lipschitz functions with constant $k$. We define a continuous function $\Phi_{k}: \mathcal{N}^{\mathbb{N}} \times \mathcal{N} \rightarrow \mathcal{N}$ that will eventually allow us to define a universal function for $\mathcal{L}_{\mathcal{N}}^{(k)}$ :

$$
\left(\Phi_{k}\left(\left(c^{(i)}\right)_{i \in \mathbb{N}}, x\right)\right)(n)=c^{(n)}\left(\tau_{k(n+1)}\left(x_{1}, x_{2}, \ldots, x_{k(n+1)-1}\right)\right) .
$$


Of course, $\Phi_{k}$ is continuous. For $\phi \in \mathcal{L}_{\mathcal{N}}^{(k)}$ we define $c_{\phi}^{(i)}: \mathbb{N} \rightarrow \mathbb{N}$ :

$$
c_{\phi}^{(i)}\left(\tau_{k(i+1)}\left(a_{1}, a_{2}, \ldots, a_{k(i+1)-1}\right)\right)=\left(\phi\left(a_{1}, a_{2}, \ldots, a_{k(i+1)-1}, 1,1, \ldots\right)\right)(i),
$$

for $\left(a_{1}, \ldots, a_{k(i+1)-1}\right) \in \mathbb{N}^{k(i+1)-1}$.

We now show that $\phi(x)=\Phi_{k}\left(\left(c_{\phi}^{(i)}\right)_{i \in \mathbb{N}}, x\right)$ for each $x \in \mathcal{N}$. Fix $x \in \mathcal{N}$ and $n \in \mathbb{N}$. Then $d\left(x,\left(x_{1}, x_{2}, \ldots, x_{k(n+1)-1}, 1,1, \ldots\right)\right) \leq 1 / k(n+1)$, and so

$$
d\left(\phi(x), \phi\left(x_{1}, x_{2}, \ldots, x_{k(n+1)-1}, 1,1, \ldots\right)\right) \leq 1 /(n+1) .
$$

Thus

$$
\begin{aligned}
(\phi(x))(n) & =\left(\phi\left(x_{1}, x_{2}, \ldots, x_{k(n+1)-1}, 1,1, \ldots\right)\right)(n) \\
& =c_{\phi}^{(n)}\left(\tau_{k(n+1)}\left(x_{1}, x_{2}, \ldots, x_{k(n+1)-1}\right)\right) \\
& =\left(\Phi_{k}\left(\left(c_{\phi}^{(i)}\right)_{i \in \mathbb{N}}, x\right)\right)(n) .
\end{aligned}
$$

Next, define $\Phi:\left(\mathbb{N} \times \mathcal{N}^{\mathbb{N}}\right) \times \mathcal{N} \rightarrow \mathcal{N}$ by

$$
\Phi\left(\left(n,\left(c^{(i)}\right)_{i \in \mathbb{N}}\right), x\right)=\Phi_{n}\left(\left(c^{(i)}\right)_{i \in \mathbb{N}}, x\right) .
$$

Note that the spaces $\mathbb{N} \times \mathcal{N}^{\mathbb{N}}$ and $\mathcal{N}$ are homeomorphic. Let $\theta: \mathcal{N} \rightarrow \mathbb{N} \times \mathcal{N}^{\mathbb{N}}$ be such a homeomorphism. Finally, define

$$
\Lambda(y, x)=\Phi(\theta(y), x),
$$

which, by construction, is universal for $\mathcal{L}_{X}$.

We use Lemma 6.3 to prove the next lemma.

Lemma 6.4. Let $\mu_{1}, \mu_{2}, \ldots \in \mathcal{C}_{\mathcal{N}}$ be arbitrary and let $\vec{i}=\left(i_{1}, i_{2}, \ldots, i_{n}\right) \in \mathbb{N}<\mathbb{N}$. Then there exists a continuous mapping $\Psi_{\vec{i}}$ universal for the family of compositions $\mathcal{A}_{\vec{i}}=\left\{\Phi_{n+1} \mu_{i_{n}} \Phi_{n} \cdots \Phi_{2} \mu_{i_{1}} \Phi_{1}: \Phi_{1}, \Phi_{2}, \ldots, \Phi_{n+1} \in \mathcal{L}_{\mathcal{N}}\right\}$.

Proof. Let $\Lambda$ be universal for $\mathcal{L}_{\mathcal{N}}$, as in Lemma 6.3 and let $\tau: \mathcal{N} \rightarrow \mathcal{N}^{n+1}$ be any homeomorphism with coordinate functions $\tau_{1}, \ldots, \tau_{n+1}$. For each $x \in \mathcal{N}$ define $\Lambda_{x}: \mathcal{N} \rightarrow \mathcal{N}$ by $\Lambda_{x}(y)=\Lambda(x, y)$. The mapping we seek is defined by

$$
\Psi_{\vec{i}}(x, y)=\Lambda_{\tau_{n+1}(x)} \mu_{i_{n}} \cdots \Lambda_{\tau_{2}(x)} \mu_{i_{1}} \Lambda_{\tau_{1}(x)}(y) .
$$

It is routine to verify that this mapping is, indeed, continuous and universal for $\mathcal{A}_{\vec{i}}$.

Proof of Theorem 6.1. Part I. In this part of the proof we will show that

$$
\operatorname{rank}\left(\mathcal{C}_{\mathcal{N}}: \mathcal{L}_{\mathcal{N}}\right) \geq \aleph_{1}
$$

i.e. $\operatorname{rank}\left(\mathcal{C}_{\mathcal{N}}: \mathcal{L}_{\mathcal{N}}\right)$ is uncountable.

Let $\mu_{1}, \mu_{2}, \ldots \in \mathcal{C}_{\mathcal{N}}$ be arbitrary and let $\vec{i}=\left(i_{1}, i_{2}, \ldots, i_{n}\right)$. Enumerate the families $\mathcal{A}_{\vec{i}}$, from Lemma 6.4 as $\mathcal{A}_{1}, \mathcal{A}_{2}, \ldots$ with universal continuous mappings $\Psi_{1}, \Psi_{2}, \ldots$. The function $\Psi: \mathcal{N} \times \mathcal{N} \rightarrow \mathcal{N}$ defined by

$$
\Psi\left(\left(n_{1}, n_{2}, \ldots\right),\left(m_{1}, m_{2}, \ldots\right)\right)=\Psi_{n_{1}}\left(\left(n_{2}, n_{3}, \ldots\right),\left(m_{1}, m_{2}, \ldots\right)\right)
$$

is continuous and universal for $\bigcup_{i=1}^{\infty} \mathcal{A}_{i}$. By Proposition 6.2, it follows that

$$
\left\langle\mathcal{L}_{\mathcal{N}}, \mu_{1}, \mu_{2}, \ldots\right\rangle=\bigcup_{i=1}^{\infty} \mathcal{A}_{i} \neq \mathcal{C}_{\mathcal{N}}
$$


Before giving the second part of the proof of Theorem 6.1 we state one further auxiliary lemma.

For a topological space $X$, we say that a function $f: X \rightarrow[0,1]$ is lower semicontinuous or upper semicontinuous if the sets $f^{-1}(y, 1], f^{-1}[0, y)$, respectively, are open in $X$ for each $y \in[0,1]$. There exists a lower semicontinuous function $L: \mathcal{N}^{2} \rightarrow[0,1]$ universal for all lower semicontinuous mappings from $\mathcal{N}^{2}$ to $[0,1]$; see, for example, 3. Equivalently, there exists an upper semicontinuous function $U: \mathcal{N}^{2} \rightarrow[0,1]$ universal for all such functions from $\mathcal{N}^{2}$ to $[0,1]$. We will construct lower and upper semicontinuous functions $L^{\prime}$ and $U^{\prime}$, respectively, that are independent and universal for all lower and upper semicontinuous functions, respectively. In other words, for any lower semicontinuous $l: \mathcal{N} \rightarrow[0,1]$ and upper semicontinuous $u: \mathcal{N} \rightarrow[0,1]$ there exists $x \in \mathcal{N}$ such that $l(\cdot)=L^{\prime}(x, \cdot)$ and $u(\cdot)=U^{\prime}(x, \cdot)$.

Let $\phi=\left(\phi_{1}, \phi_{2}\right): \mathcal{N} \rightarrow \mathcal{N}^{2}$ be a homeomorphism and define $\theta_{1}(x, y)=\left(\phi_{1}(x), y\right)$ and $\theta_{2}(x, y)=\left(\phi_{2}(x), y\right)$. Of course, $\theta_{1}$ and $\theta_{2}$ are continuous. Now, define $L^{\prime}$ : $\mathcal{N}^{2} \rightarrow[0,1]$ by

$$
L^{\prime}(x, y)=L\left(\phi_{1}(x), y\right)=L \circ \theta_{1}(x, y)
$$

and define $U^{\prime}: \mathcal{N}^{2} \rightarrow[0,1]$ by

$$
U^{\prime}(x, y)=U\left(\phi_{2}(x), y\right)=U \circ \theta_{2}(x, y) .
$$

It is evident that the functions $L^{\prime}$ and $U^{\prime}$ are lower and upper semicontinuous and universal for all lower and upper semicontinuous functions, respectively, from $\mathcal{N}$ to $[0,1]$.

Lemma 6.5. There exists a continuous $\Gamma: \mathfrak{U} \rightarrow \mathcal{N}$, where $\mathfrak{U}$ is a Borel set in $\mathcal{N}^{2}$, that is universal for $\mathcal{C}_{\mathcal{N}}$.

Proof. The mappings $L^{\prime}$ and $U^{\prime}$ are defined above. Let

$$
\mathfrak{U}=\left\{(x, y): L^{\prime}(x, y)=U^{\prime}(x, y)\right\} \cap L^{\prime-1}([0,1] \backslash \mathbb{Q}) .
$$

As mentioned above, there exist homeomorphisms from $\mathcal{N}$ to the irrationals and thus also to $[0,1] \backslash \mathbb{Q}$; let $\eta:[0,1] \backslash \mathbb{Q} \rightarrow \mathcal{N}$ be one such homeomorphism. Define $\Gamma: \mathfrak{U} \rightarrow \mathcal{N}$ by

$$
\Gamma=\left.\eta \circ L^{\prime}\right|_{\mathfrak{U}}
$$

We will prove that $\Gamma$ is continuous and universal for $\mathcal{C}_{\mathcal{N}}$.

First, $L^{\prime}$ coincides with $U^{\prime}$ on $\mathfrak{U}$. Thus $L^{\prime}$ is lower and upper semicontinuous on $\mathfrak{U}$ and so is continuous on $\mathfrak{U}$. Hence $\Gamma$ is continuous on $\mathfrak{U}$.

Second, let $\mu \in \mathcal{C}_{\mathcal{N}}$ be arbitrary, and define $\mu^{\prime}: \mathcal{N} \rightarrow[0,1] \backslash \mathbb{Q}$ by $\mu^{\prime}(y)=\eta^{-1} \circ$ $\mu(y)$. Note that $\mu^{\prime}$ is continuous and thus both upper and lower semicontinuous. By the construction of $L^{\prime}$ and $U^{\prime}$ there exists $x \in \mathcal{N}$ such that $L^{\prime}(x, \cdot)=\mu^{\prime}(\cdot)$ and $U^{\prime}(x, \cdot)=\mu^{\prime}(\cdot)$. Now, $\{x\} \times \mathcal{N} \subseteq \mathfrak{U}$, since $\mu^{\prime}$ has domain $\mathcal{N}$ and by the definition of $\mathfrak{U}$. But then

$$
\Gamma(x, \cdot)=\eta \circ L^{\prime}(x, \cdot)=\eta \circ \mu^{\prime}(\cdot)=\mu(\cdot),
$$

as required. 
We may now conclude the proof of Theorem 6.1.

Proof of Theorem 6.1. Part II. In this part of the proof we will show that

$$
\operatorname{rank}\left(\mathcal{C}_{\mathcal{N}}: \mathcal{L}_{\mathcal{N}}\right) \leq \aleph_{1}
$$

i.e. that we may find $\aleph_{1}$ mappings such that together with $\mathcal{L}_{\mathcal{N}}$ they generate $\mathcal{C}_{\mathcal{N}}$.

Let $\mathfrak{V}=\{x \in \mathcal{N}:\{x\} \times \mathcal{N} \subseteq \mathfrak{U}\}$ with the set $\mathfrak{U}$ defined in the proof of Lemma 6.5. The set $\mathfrak{V}$ is coanalytic. Therefore by [10, Section 39, II, Cor. 3], $\mathfrak{V}$ is the union of $\aleph_{1}$ nonempty Borel sets $B_{\alpha}, \alpha<\aleph_{1}$. Moreover, by [10, Section 37, I, Th. 1] for each $\alpha<\aleph_{1}$ there exists a continuous surjective mapping $\mu_{\alpha}$ from $\mathcal{N}$ onto $B_{\alpha}$.

Let $\sigma: \mathcal{N} \rightarrow \mathcal{N}^{2}$ be a Lipschitz homeomorphism, the existence of which is discussed at the beginning of this section. Define $\Phi_{\alpha}: \mathcal{N} \rightarrow \mathcal{N}$ by

$$
\Phi_{\alpha}\left(\sigma^{-1}(x, z)\right)=\Gamma\left(\mu_{\alpha}(x), z\right),
$$

with $\Gamma$ defined as in Lemma 6.5

Let $\tau \in \mathcal{C}_{\mathcal{N}}$ be arbitrary. We will prove that $\tau$ is the composition of a Lipschitz mapping and $\Phi_{\alpha}$ for some $\alpha$. Since $\Gamma$ is universal for $\mathcal{C}_{\mathcal{N}}$ there exists $y \in \mathcal{N}$ such that $\tau(\cdot)=\Gamma(y, \cdot)$. In fact, $\{y\} \times \mathcal{N} \subseteq \mathfrak{U}$, and so $y \in \mathfrak{V}$. In particular, $y \in B_{\alpha}$ for some $\alpha<\aleph_{1}$. Define $\Lambda: \mathcal{N} \rightarrow \mathcal{N}$ such that $\Lambda(z)=\sigma^{-1}(x, z)$, where $\mu_{\alpha}(x)=y$. Since $z \mapsto(x, z)$ is Lipschitz and $\sigma^{-1}$ is Lipschitz, so is $\Lambda$.

To conclude, for any $z \in \mathcal{N}$ we have

$$
\tau(z)=\Gamma(y, z)=\Gamma\left(\mu_{\alpha}(x), z\right)=\Phi_{\alpha}\left(\sigma^{-1}(x, z)\right)=\Phi_{\alpha} \circ \Lambda(z) .
$$

\section{REFERENCES}

1. S. Banach, Sur un theorème de M. Sierpiński, Fund. Math. 25 (1935) 5-6.

2. H. Cook, Continua which admit only the identity mapping onto non-degenerate subcontinua, Fund. Math. 60 (1967) 241-249. MR0220249 (36:3315)

3. J. Cichoń and M. Morayne, Universal functions and generalized classes of functions, Proc. American Math. Soc. 102 (1988) 83-89. MR0915721 (89c:26003)

4. R. Engelking, On closed images of the space of irrationals, Proc. Amer. Math. Soc. 21 (1969) 583-586. MR0239571 (39:928)

5. F. Galvin, Generating countable sets of permutations, J. London Math. Soc. 51 (1995) 230242. MR.1325568 (96a:20005)

6. P.M. Higgins, J.M. Howie and N. Ruškuc, Generators and factorisations of transformation semigroups, Proc. Roy. Soc. Edinburgh Sect. A 128 (1998) 1355-1369. MR1663980 (2000a:20139)

7. P.M. Higgins, J.M. Howie, J.D. Mitchell and N. Ruškuc, Countable versus uncountable ranks in infinite semigroups of transformations and relations, Proc. Edinburgh Math. Soc. 46 (2003) 531-544. MR2013951 (2004h:20093)

8. P. M. Higgins, J. D. Mitchell, M. Morayne and N. Ruškuc, Rank properties of endomorphisms of infinite partially ordered sets, Bull. London Math. Soc. 38 (2006) 177-191. MR2214470

9. J.M. Howie, N. Ruškuc and P. Higgins, On relative ranks of full transformation semigroups, Comm. Algebra 26 (1998) 733-748. MR1606221 (99b:20099)

10. K. Kuratowski, Topology, Vol. I, Academic Press, New York, London; Państwowe Wydawnictwo Naukowe, Warsaw 1966. MR0217751 (36:840)

11. T. Maćkowiak, Singular arc-like continua, Dissertationes Math. (Rozprawy Mat.) 257 (1986). MR0881290 (88f:54066)

12. W. Sierpiński, Sur les suites infinies de fonctions définies dans les ensembles quelconques, Fund. Math. 24 (1935) 209-212. 
13. S. Subbiah, Some finitely generated subsemigroups of $S(X)$, Fund. Math. 86 (1975) 221-231. MR0405383 (53:9177)

14. H. Vaughan, On locally compact metrisable spaces, Bull. Amer. Math. Soc, 43 (1937) 532535.

Institute of Mathematics, WrocŁaw University of Technology, Wybrzeże WyspiańSkiego 27, 50-370 WrocŁaW, Poland

E-mail address: Jacek.Cichon@pwr.wroc.pl

Mathematics Institute, University of St Andrews, North Haugh, St Andrews, Fife, KY16 9SS, SCOTLAND

E-mail address: jdm3@st-and.ac.uk

Institute of Mathematics and Computer Science, Wroceaw University of Technology, Wybrzeże Wyspiańskiego 27, 50-370 Wroceaw, Poland

E-mail address: Michal.Morayne@pwr.wroc.pl 